\title{
Optimized Reconstruction Algorithm-Processed CT Image in the Diagnosis of Correlation between Epicardial Fat Volume and Coronary Heart Disease
}

\author{
Enzhong Xue $\mathbb{D}$ and Qiangqiang Jing \\ Department of Cardiovascular Medicine, Yan'an People's Hospital, Yan'an 716000, Shaanxi, China \\ Correspondence should be addressed to Qiangqiang Jing; 1923131201@siit.edu.cn
}

Received 6 October 2021; Revised 25 November 2021; Accepted 20 December 2021; Published 6 January 2022

Academic Editor: M Pallikonda Rajasekaran

Copyright ( 2022 Enzhong Xue and Qiangqiang Jing. This is an open access article distributed under the Creative Commons Attribution License, which permits unrestricted use, distribution, and reproduction in any medium, provided the original work is properly cited.

\begin{abstract}
This study was to analyze the application value of a reconstruction algorithm in CT images of patients with coronary heart disease and analyze the correlation between epicardial fat volume and coronary heart disease. An optimized reconstruction algorithm was constructed based on compressed sensing theory in this study. Then, the optimized algorithm was applied to the image reconstruction of multislice spiral CT image data after testing its sensitivity, accuracy, and specificity. 60 patients with suspected angina pectoris were divided into lesion group (40 cases) and normal group (20 cases) according to whether there were coronary atherosclerotic plaques in cardiac vessels. The results showed that the sensitivity, specificity, and accuracy of the optimized reconstruction algorithm were $91.78 \%, 84.27 \%$, and $95.32 \%$, and the running time was $(12.18 \pm 2.49) \mathrm{s}$. The CT value of the liver and the CT ratio of the liver and spleen in the lesion group were $(53.81 \pm 5.91)$ and $(3.88 \pm 0.67)$, respectively. There was no significant difference between the two groups $(P>0.05)$. The body mass index and epicardial fat volume in the lesion group were $(31.93 \pm 4.54) \mathrm{kg} / \mathrm{m}^{2}$ and $(120.09 \pm 22.01) \mathrm{cm}^{3}$, respectively. The body mass index and fat volume in the lesion group were significantly higher than those in the normal group $(P<0.05)$. The epicardial fat constitution increased with the increase of the number of coronary arteries involved, and there was a positive correlation between them. Among patients with different coronary atherosclerotic plaques, the epicardial fat volume in patients with mixed plaques was the largest $(P<0.05)$. In summary, optimizing CT images under compressed a sensing reconstruction algorithm could effectively improve the diagnostic accuracy of doctors. Epicardial fat volume was positively correlated with coronary heart disease. Epicardial fat volume could be used as one of the important indexes to predict coronary heart disease.
\end{abstract}

\section{Introduction}

Coronary atherosclerotic heart disease is also known as coronary heart disease. It is the general term for a series of cardiovascular diseases arising from coronary atherosclerotic plaques, causing insufficient local blood supply and insufficient oxygen supply to the heart. It has gradually become a major disease threatening human life [1]. Studies have shown that the patients with coronary heart disease are younger, from senior patients to middle-aged, and even young people.

Epicardial fat is visceral fat deposited around the heart. It can buffer the torsion of the coronary arteries caused by arterial pulsation and heart contraction, promote the remodeling of the coronary arteries, and regulate the myocardium and coronary arteries through paracrine. It affects the metabolism of the myocardium and coronary artery smooth muscle and then influences the heart or vascular functions [2]. Clinical evidence shows that epicardial fat or paracoronary fat is closely related to coronary atherosclerosis [3]. Also, epicardial fat can be used as a marker to reflect the local metabolism because it is closely related to traditional vascular risk factors and can reflect the progress of coronary atherosclerosis from the perspective of local inflammation and system metabolism [4]. 
As for the correlation between myocardial fat and coronary heart disease, accurate quantification of myocardial fat is essential. Studies confirmed that myocardial fat was closely related to visceral fat but had no significant correlation with overall fat $[5,6]$. Other indicators measured on the surface of the fat body, such as waist circumference (WC) and hip circumference (HC), reflected the fat distribution, but there were great errors, and they could not well predict the amount of fat. Therefore, the direct evaluation methods of pericardial fat such as ultrasound, MRI, and MSCT were preferred. Ultrasound was simple and cheap, but its accuracy and repetition accuracy were poor. At present, it is unable to fully evaluate the total amount of myocardial adipose tissue. MRI and MSCT were considered as the "gold standard" for diagnosis, but MRI imaging was time-consuming and costly. MSCT had short scanning time and was widely used [7]. Image reconstruction technology played an important role in many fields. In the research and implementation of the reconstruction algorithm, there were a series of extremely complex image processing problems and mathematical calculation problems. At present, algorithms such as image reconstruction and computer-aided medical image analysis have obvious advantages in technical breakthroughs and improve the medical level and have also become an effective way to solve medical image problems. The optimized iterative reconstruction of a CT image is an alternative image reconstruction method. It is a process based on the CT detector receiving the original data that can characterize the X-ray intensity, solving the pixel values in the image matrix, and restoring the human anatomical structure. It can image under low radiation [8]. The optimized reconstruction algorithm can fit the local structure model of image data and greatly reduce irrelevant noise [9]. Therefore, this study will use the optimized reconstruction algorithm to reconstruct and analyze the multislice spiral CT images so as to improve the quality of CT images and the effect of auxiliary diagnosis and treatment.

In the study, the compressed sensing-based reconstruction algorithm was used to process the multislice spiral CT (MSCT) images to lift the diagnosis rate. The correlation between the volume of epicardial fat and coronary heart disease was discussed then, aiming to find a new method to predict coronary heart disease.

\section{Materials and Methods}

2.1. Research Subjects. In this study, 60 patients with suspected angina pectoris, including 41 males and 19 females, with an average age of $(55.3 \pm 4.17)$ years, admitted to the hospital from January 20, 2019, to February 15, 2020, were selected as research subjects. They were diagnosed by two doctors who have been engaged in coronary CTA image diagnosis for more than 5 years. They all underwent MSCT for coronary artery, and the CT images were processed by the optimized iterative reconstruction algorithm. According to whether there were coronary atherosclerotic plaques in the cardiac vessels, patients were divided into the pathological group $(n=40)$ and the normal group $(n=20)$. The study has been approved by the ethics committee of the hospital, and the patients and their families understood the study and signed an informed consent form.

Inclusion criteria were defined as follows: (1) patients aged between 45 and 65; (2) patients with clear consciousness and can cooperate with diagnosis and treatment; (3) patients with complete clinical data and information; and (4) patients with no history of mental illness and emotional stability.

Exclusion criteria were defined as follows: patients with excessive respiratory amplitude during MSCT scanning and sudden change of heart rate during scanning, resulting in large artifacts or large level changes.

\subsection{Multislice Spiral CT Coronary Angiography.}

Preoperative examination: preparation of patients before examination: patients must fast six hours before the examination, and Betaloc was taken to control the heart rate below 70 beats/min. Breathing training was received [10], breathing was kept within 22 seconds after breathing, and multiple training was received until all the patients understand and master it. Attention was paid to keeping the same amplitude of each inhalation. The examination process of the patient was briefly described before the examination to avoid respiratory tract movement and heart rate fluctuation caused by tension or complaint.

LightSpeed 16 MSCT was used to scan. The layer thickness was $0.425 \mathrm{~mm}$, the spiral distance was 0.701 , the frame rate was $0.5 \mathrm{~s} /$ turn, the voltage was $100 \sim 150 \mathrm{kV}$, the current was 300 500 $\mathrm{mA}$, the field of view was $250 \mathrm{~mm}$, and the matrix was $498 \times 498$. The cardiac scanning mode and retrospective ECG analysis were selected. According to the patient's heart rate, single-, double-, and four-slice scanning were adopted, namely, the heart rate was $50 \sim 60$ beats $/ \mathrm{min}$, with a small range. Single-sector scanning (snapshot segment) was chosen, when the heart rate was $65 \sim 75$ beats $/ \mathrm{min}$. Double-sector scanning (snapshot burst), large-scale scanning, or four-sector scanning (snapshot burst plus) were chosen, when the heart rate was $>75$ beats $/ \mathrm{min}$. $320 \mathrm{ml}$ or $400 \mathrm{mg} / \mathrm{L}$ nonionic contrast medium (Onepak or Ultravist) was injected into the anterior elbow vein [11], and the flow rate was $4 \sim 5 \mathrm{ml} / \mathrm{s}$. The aortic root was scanned continuously with a delay of eight seconds and a scanning cycle of two seconds. The region of interest of the aortic root was taken, and the time density curve was obtained. The peak time of contrast center concentration was used as the scanning delay time.

$80 \sim 120 \mathrm{~mL}$ of nonionic contrast agent was injected at a flow rate of $4 \sim 5 \mathrm{~mL} / \mathrm{s}$. After the contrast agent passed through the anterior elbow vein, the doctor started scanning according to the predetermined delay time, and the patient hold his breath for 15 25 seconds after a deep inhalation. The reconstruction thickness of the original data was $0.425 \mathrm{~mm}$, and the reconstruction interval was $0.527 \mathrm{~mm}$. The standard algorithm was used to reconstruct the original data after $20 \%, 40 \%, 60 \%$, and $80 \%$ of the phase behind the $\mathrm{R}$ wave in the $\mathrm{R}-\mathrm{R}$ interval. The horizontal axis images reconstructed in each stage were transmitted to GE AW4.3 workstation for analysis and research and observed through 
multiplanar reconstruction (MPR), maximum density projection (MIP), curved plane reconstruction (CPR), and volume reconstruction (VR). The best image phase window was selected for the imaging evaluation of cardiac vascular diameter (Figure 1).

\subsection{Evaluation and Analysis of Coronary Arteries. The MSCT} images for the coronary artery were transmitted to the AW4.3 workstation for analysis. The coronary artery of each patient was divided into 13 segments, and two senior doctors were responsible for diagnosis as per the coronary artery segmentation method by the American Heart Association [12]. Any inconsistencies were solved by discussion. Cardiac catheters with a tube diameter of $\geq 2 \mathrm{~mm}$ were evaluated. Coronary artery stenosis can be divided into three categories: (i) no arteriosclerosis, (ii) nonobstructive arteriosclerosis (lumen stenosis <50\%), and (iii) obstructive arteriosclerosis (lumen stenosis $\geq 50 \%$ ) (Figure 2).

2.4. The Epicardial Fat Volume. GE LightSpeed 16 MSCT produced in the United States was used for retrospective scanning. The scanning thickness of the instrument is $3 \mathrm{~mm}$, the voltage is 120 to $140 \mathrm{kV}$, the current is $280 \sim 350 \mathrm{~mA}$, the field of view is $250 \mathrm{~mm}$, and the matrix is $490 \times 490$. During the scan, the patient was instructed to hold his breath until the scan was over to minimize respiratory infections. After scanning, the Volume Viewer was used to measure the fat volume on the semiautomatic offline workstation [13]. The specific method was as follows: the operator manually tracked the pericardium, separated the heart, and harvested fat with a window width of 250-30 HU, followed by volume measurement (Figure 3).

2.5. The Optimized Compressed Sensing CT Reconstruction Algorithm. Compressed sensing (CS) is a new theory different from traditional data processing technologies. With the major theoretical breakthrough made by Donoho in 2006 as a hallmark, compressed sensing theory is marching forward continuously [14]. Its basic theoretical system equation is as follows:

$$
y \in A^{k}
$$

Equation (1) can also be expressed as follows:

$$
y=\psi \ell,
$$

where $\psi$ represents the basis or sparse basis, $\ell$ represents the coefficient, and $\|\ell\|_{0} \leq N$ represents that the signal is an $N$ sparse signal.

In the observation array,

$$
\vartheta \in A^{n \times k} \text {. }
$$

If $n>k$, the observation data of the signal are acquired as follows:

$$
X=\vartheta y .
$$

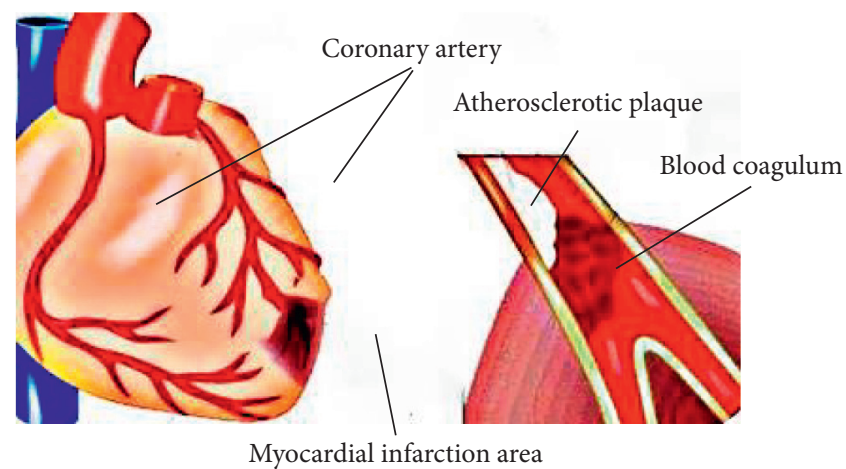

FIgURE 1: Schematic diagram of coronary atherosclerosis.

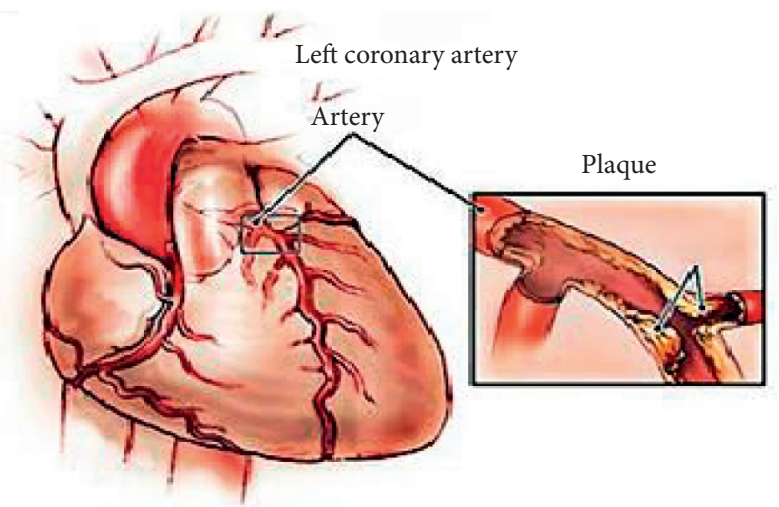

FIGURE 2: Schematic diagram of the coronary plaque.

Assume that the observation array can meet the restricted isometry property (RIP), and then, the signal $y \in A^{k}$ can be accurately recovered from the compressed observation data $x \in A^{k}$. When the observation matrix is a Gaussian random matrix or other matrix, the lower limit of $n$ is as follows:

$$
n>A B \log \left(\frac{k}{p}\right) \text {, }
$$

where $A$ represents a positive constant. In general, since $n<k$, it is not feasible to recover $y$ from $x$. However, when a certain condition is reached, that is, when $y$ meets the requirements of sparsity and RIP of the observation array, the signal can be restored perfectly through nonlinear optimization, and the equation is as follows:

$$
\begin{aligned}
& \hat{\wp}=\arg \min \|\ell\|_{0}, g \cdot y \cdot e=\vartheta \psi \ell, \\
& \hat{y}=\psi \hat{\ell}
\end{aligned}
$$

where $\hat{y}$ is the estimated value of $y$ and $\hat{\ell}$ is the estimated value of $\ell$.

Under incomplete projection data, traditional reconstruction algorithms cannot meet the actual clinical requirements in terms of reconstruction speed and reconstruction accuracy. Compressed sensing theory can provide new research ideas for the reconstruction of incomplete projection data [15]. First of all, the compressed 


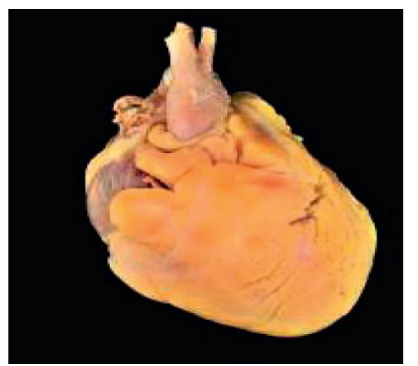

(a)

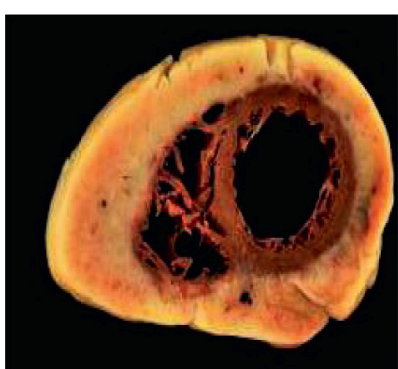

(b)

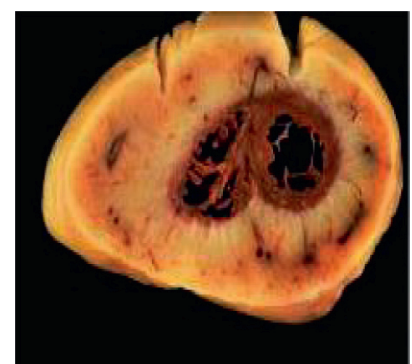

(c)

FIgURE 3: The epicardial fat deposition. (a) Diagram of the epicardial fat coating of the heart; (b) schematic diagram of the upper horizontal plane of the epicardium; (c) schematic diagram of the lower horizontal plane of the epicardium.

sensing theory does not require the location of information collection, and this theory does not require the amount of image information collected. It can reconstruct CT images based on incomplete data. The construction process is as follows:

$$
\hat{y}=\arg \min \|y\|_{0}, g \cdot y \cdot A=W y,
$$

where $\hat{y}$ requires the estimated value of image $y, W$ is the projection coefficient matrix, and $A$ is the projection data. Then, this equation is converted into an optimization problem, and the equation is as follows:

$$
\min _{y} \underset{\text { data fidelity }}{f(y)}+\underset{\text { regularizer }}{\gamma m(y),}
$$

where $f(y)$ is the fidelity of the data, which represents the difference between the reconstructed CT image and the real image. In clinical applications, most of the real images are unknowable, and it is estimated per deviation between forward projection of the reconstructed image and the measured value.

$$
f(y)=\|B \hat{y}-A\|^{t},
$$

where \|\|$^{t}$ is the $t$-norm. When $t$ takes different values, different standards are used to measure the fidelity of the data. In the study, the more common Euclidean distance is adopted when $t=2$. Based on the theory of compressed sensing, applying iterative algorithms in the CT reconstruction process can effectively reduce the dose and angle of CT scanning, which should be suggested.

2.6. Evaluation Indicators. In this study, the optimized reconstruction algorithm is evaluated factoring into the accuracy, sensitivity, and specificity, and the specific equations are as follows:

$$
\begin{aligned}
& A=\frac{F_{T}}{T} \times 100 \%, \\
& S=\frac{P}{W+F} \times 100 \%, \\
& Y=\frac{N}{W+F} \times 100 \%,
\end{aligned}
$$

where $A$ is the accuracy, $S$ is the sensitivity, and $Y$ is the specificity; $F_{T}$ indicates the number of accurate predictions, $T$ indicates the total number of patients, $T$ indicates the true positive, $N$ indicates true negative, $F$ indicates false positive, and $W$ means false negative.

2.7. Statistical Methods. The data were processed by SPSS19.0. Measurement data were expressed as mean\pm standard deviation $(\bar{x} \pm s)$, and count data were expressed as a percentage. The $t$ test and $\chi 2$ test were performed. The pathological group and the normal group were compared for the liver and spleen CT ratio, body mass index, epicardial fat, coronary artery lesions, and plaque types. The variance test was used between the groups, and $P<0.05$ was the threshold for significance.

\section{Results}

3.1. Classification Performance of the Hybrid Iterative Algorithm. Figure 4 shows the sensitivity, specificity, accuracy, and running time of the compressed sensing-based iterative reconstruction algorithm. It was noted that the sensitivity was $91.78 \%$, the specificity was $84.27 \%$, the accuracy rate was $95.32 \%$, and the running time was $(12.18 \pm 2.49) \mathrm{s}$, suggesting that the compressed sensingbased reconstruction algorithm lifted the diagnosis accuracy.

3.2. The General Data of Patients. A total of 60 inpatients in the cardiology department were selected in this study, including 41 males (68.33\%) and 19 females (31.67\%) (Figure 5), with an average age of $(55.3 \pm 4.17)$ years. According to the presence or absence of atherosclerotic plaque in the coronary artery, forty cases were chosen in the lesion group, including 30 males and 10 females with an atherosclerotic plaque on coronary angiography. The mean age of patients in the lesion group was $(54.21 \pm 3.89)$ years. Twenty cases were chosen in the normal group, including 11 males and 9 females with no abnormality in coronary angiography. The average age of patients in the normal group was $(55.14 \pm 5.79)$ years (Figure 6). Figure 7 shows a CT image of a patient with coronary heart disease. 


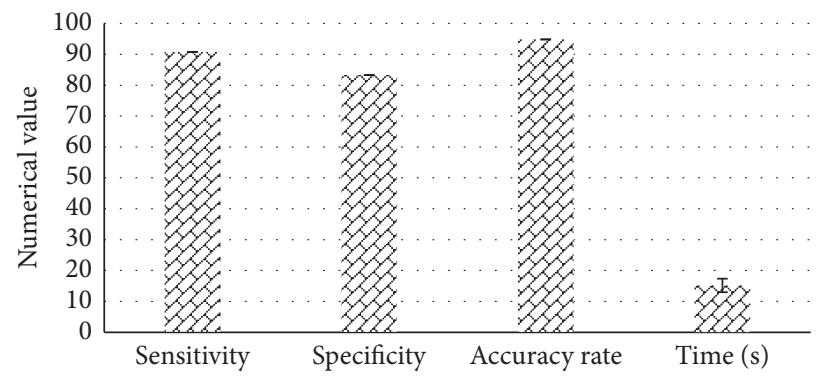

FIGURE 4: The accuracy, sensitivity, specificity, and running time of the hybrid iterative algorithm.

$68.33 \%$

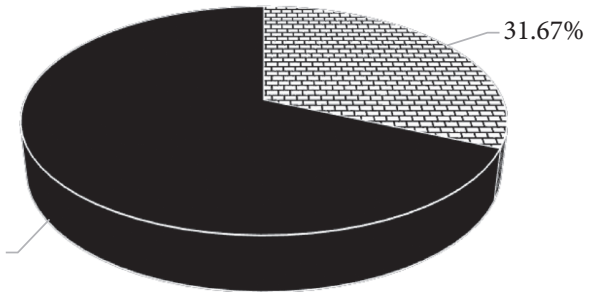

意 Women

Men

Figure 5: The male-to-female ratio.

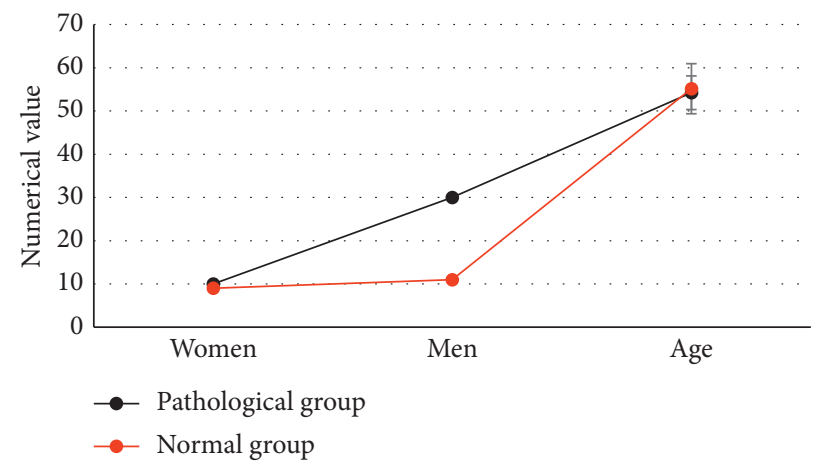

FIGURE 6: The general information of the two groups of patients.

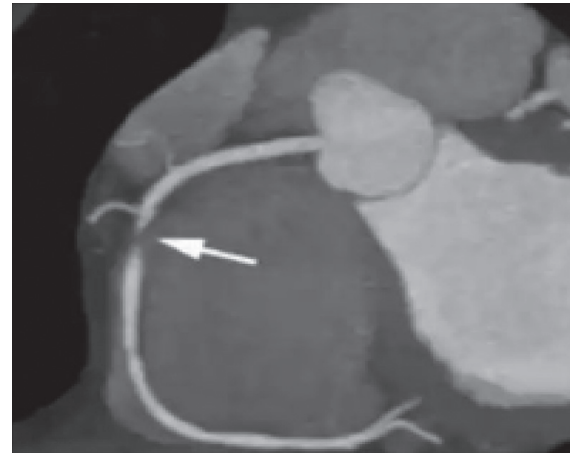

(a)

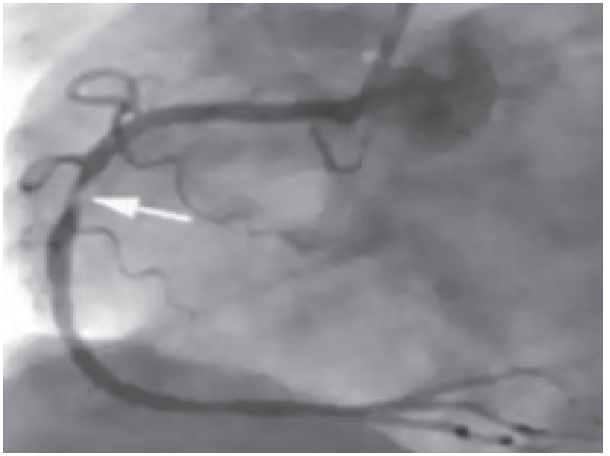

(b)

FIgURE 7: A CT image of a patient with coronary heart disease. (a) The coronary CT results; (b) the coronary angiography results, both showing more than $90 \%$ vascular stenosis. 
3.3. Comparison of Indicators between the Two Groups. Figure 8 shows the liver CT value, liver CT-to-spleen CT ratio, body mass index, and epicardial fat volume of the two groups of patients. It was noted that the liver CT value and liver CT-to-spleen CT ratio of the pathological group were $(53.81 \pm 5.91)$ and $(3.88 \pm 0.67)$, respectively. The corresponding values in the normal group were $(52.19 \pm 4.92)$ and $(3.91 \pm 0.72)$, respectively. There were no notable differences between the two groups $(P>0.062)$; the body mass index and epicardial fat volume of the pathological group were $(31.93 \pm 4.54) \mathrm{kg} / \mathrm{m}^{2}$ and $(120.09 \pm 22.01) \mathrm{cm}^{3}$, respectively, and those in the normal group were $(20.01 \pm 2.91) \mathrm{kg} / \mathrm{m}^{2}$ and $(70.93 \pm 16.82) \mathrm{cm}^{3}$. Obviously, the body mass index and fat volume of the pathological group were larger than those of the normal group, and there were notable differences between the groups $(P<0.05)$.

3.4. Correlation between Coronary Artery Involvement Index and Epicardial Fat Volume in the Pathological Group. In the pathological group, 30 patients with moderate or severe stenosis in the main coronary artery were selected. They were regrouped according to the number of branches of the coronary artery involved in the disease, and the correlation between the number of branches and the body mass index and epicardial fat volume was analyzed. The results are shown in Figure 9. There were 11 cases with 1 branch involved, 9 cases with 2 branches involved, and 10 cases with 3 branches involved, and the volumes of epicardial fat were $(95.18 \pm 10.72) \quad \mathrm{cm}^{3}, \quad(148.27 \pm 17.25) \quad \mathrm{cm}^{3}, \quad$ and $(180.72 \pm 20.32) \mathrm{cm}^{3}$, respectively. Obviously, with the increase in the number of branches involved, the epicardial fat volume also increased, and the two were positively correlated. There was no obvious correlation between the body mass index and the coronary artery involvement index.

3.5. Correlation between Coronary Artery Disease and the Volume of Epicardial Fat in the Pathological Group. Of the 40 patients in the pathological group, there were 12 patients with calcified plaques, 12 patients with noncalcified plaques, and 16 with mixed plaques. The epicardial fat volumes were $(88.27 \pm 6.82),(119.83 \pm 6.92)$, and $(168.92 \pm 11.82)$, respectively. Obviously, the epicardial fat volume of patients with mixed plaques was the largest $(P<0.05)$. No notable differences were noted in the body mass index of patients with different types of plaques $(P>0.05)$ (Figure 10).

\section{Discussion}

Coronary heart disease is a deadly killer endangering human life and health, and there is an increasing number of patients suffering from it. There are more and more studies on the risk factors of coronary heart disease. Before, obesity has been considered to be an important cause of coronary heart disease. Studies of Langet et al. [14] showed that the inflammatory cells produced by various adipose tissues in the human body changed the function of vascular wall endothelial cells, smoothed muscle tissue, and other cells, resulting in the production of atherosclerotic plaque. Based

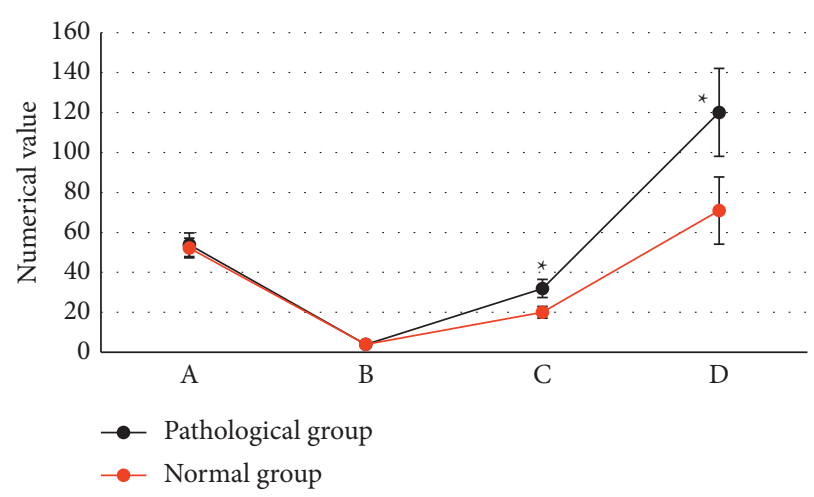

FIgURE 8: The fat volume and body mass index in the two groups of patients. ${ }^{*}$ indicates that the pathological group was notably different from the normal group $(P<0.05)$. A: the liver $\mathrm{CT}$ value; $\mathrm{B}$ : the liver and spleen CT values; $C$ : the body mass index; $D$ : the epicardial fat volume.

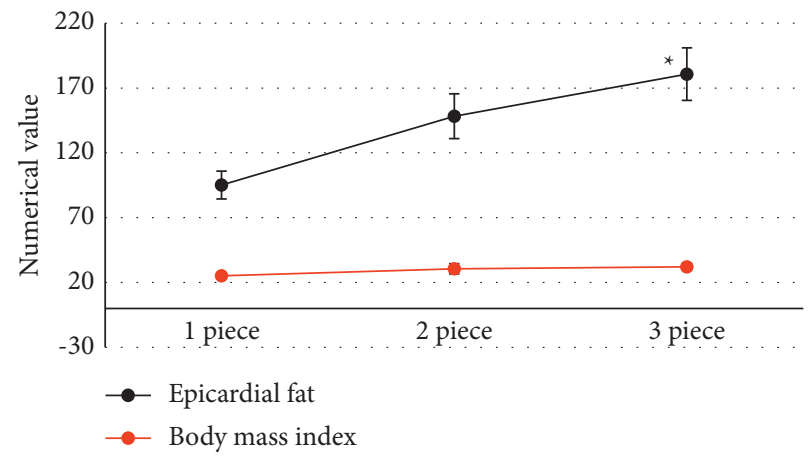

FIgURE 9: Correlation between the patient's body mass index and epicardial fat volume and the number of coronary artery branches involved. ${ }^{*}$ indicates a notable difference versus those with 3 branches involved $(P<0.05)$.

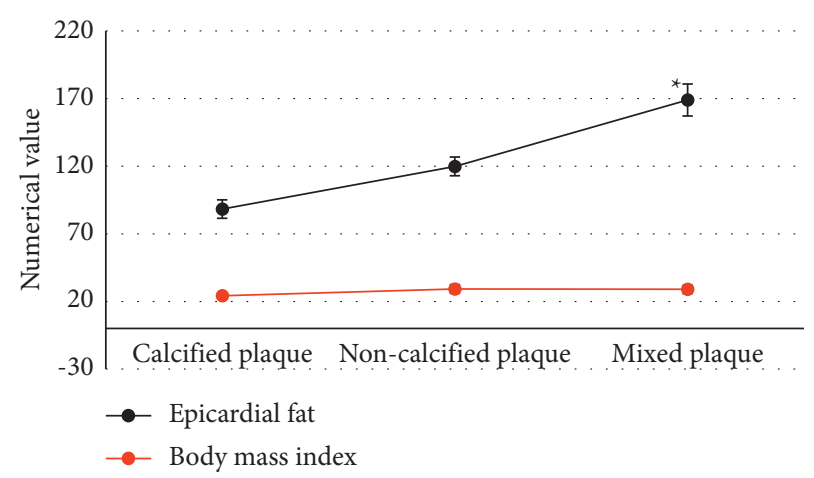

FIgURE 10: The epicardial fat volume of patients with different types pf plaques. ${ }^{*}$ indicates a notable difference versus the patients with mixed plaques $(P<0.05)$.

on the theory of compressed sensing, an optimized reconstruction algorithm was constructed firstly, and the algorithm in the diagnosis of the CT image was used. Then, the correlation between epicardial fat volume and coronary heart disease was studied and discussed. The results show that the sensitivity, specificity, and accuracy of the optimized 
compressed sensing iterative reconstruction algorithm were $91.78 \%, 84.27 \%$, and $95.32 \%$, and the running time was $(12.18 \pm 2.49)$ s. It was found that the sensitivity, specificity, and accuracy of the algorithm were high, indicating that the optimized reconstruction algorithm was of great help to improve the accuracy of CT image diagnosis. Moreover, it helped doctors assist in diagnosis, which greatly improved the diagnosis efficiency of the disease. The research results were highly similar to the research conclusions of Nagayama et al. [16]. It indicated that the application of the optimized algorithm to CT image diagnosis could improve the accuracy of disease diagnosis. The research of Yin et al. [8] also suggested that the CT processing software optimized by the algorithm had high accuracy in measuring epicardial adipose tissue. In this research topic, it also revealed that the CT image measurement technology optimized and reconstructed had better performance and could reduce some economic pressure for patients.

The liver CT value and liver CT-to-spleen CT ratio of the pathological group were $(53.81 \pm 5.91)$ and $(3.88 \pm 0.67)$, respectively. The corresponding values in the normal group were $(52.19 \pm 4.92)$ and $(3.91 \pm 0.72)$, respectively. There were no notable differences between the two groups $(P>0.05)$; the body mass index and epicardial fat volume of the pathological group were $(31.93 \pm 4.54) \mathrm{kg} / \mathrm{m}^{2}$ and $(120.09 \pm 22.01) \mathrm{cm}^{3}$, respectively, and those in the normal group were $(20.01 \pm 2.91) \mathrm{kg} / \mathrm{m}^{2}$ and $(70.93 \pm 16.82) \mathrm{cm}^{3}$. Obviously, the body mass index and fat volume of the pathological group were larger than those of the normal group, and there were notable differences between the groups $(P<0.05)$. There were 11 cases with 1 branch involved, 9 cases with 2 branches involved, and 10 cases with 3 branches involved, and the volumes of epicardial fat were $(95.18 \pm 10.72) \quad \mathrm{cm}^{3}, \quad(148.27 \pm 17.25) \quad \mathrm{cm}^{3}, \quad$ and $(180.72 \pm 20.32) \mathrm{cm}^{3}$, respectively. Obviously, with the increase in the number of branches involved, the epicardial fat volume also increased, and the two were positively correlated. There was no obvious correlation between body mass index and coronary artery involvement index. Milanese et al. [17] found that the degree of infiltration in the liver and spleen was not obviously associated with the development of coronary atherosclerosis, but the volume of epicardial fat was directly associated with the occurrence of coronary heart disease. This was consistent with the results of this study, indicating that the volume of epicardial fat was positively correlated with the occurrence of coronary heart disease. Of the 40 patients in the pathological group, there were 12 patients with calcified plaques, 12 patients with noncalcified plaques, and 16 with mixed plaques. The epicardial fat volumes were $(88.27 \pm 6.82) \mathrm{cm}^{3},(119.83 \pm 6.92) \mathrm{cm}^{3}$, and $(168.92 \pm 11.82) \mathrm{cm}^{3}$, respectively. Obviously, the epicardial fat volume of patients with mixed plaques was the largest $(P<0.05)$. No notable differences were noted in the body mass index of patients with different plaque types $(P>0.05)$. Kim et al. [18] showed that epicardial fat was a biochemically active tissue that supplied coronary artery blood together with cardiomyocytes, and there was no fascia separating epicardial fat tissue from cardiomyocytes. Compared to the body mass index relating to fat in the whole body, epicardial adipose tissue can better illustrate the correlation with coronary heart disease. Notable differences were noted in the volume of epicardial fat between patients with different plaques types, suggesting that coronary heart disease can be forecast through epicardial fat volume and that it has a higher clinical application value compared with indicators such as the body mass index and waist circumference.

\section{Conclusion}

Firstly, an optimized reconstruction algorithm is constructed based on compressed sensing theory. After testing its sensitivity, accuracy, and specificity, it is applied to the image reconstruction of CT image data. The results show that optimizing the CT image under the compressed sensing reconstruction algorithm can effectively improve the diagnostic accuracy of doctors. Epicardial fat volume is positively correlated with coronary heart disease. Epicardial fat volume can be used as one of the important indexes to predict coronary heart disease. However, there are still some deficiencies in this study. This study lacks the control test of other algorithms in the experimental process. Therefore, the experimental results are subjective. In addition, there are few research samples, and the test results are not suitable for large-scale use. In future work, further comparative experiments will be conducted to fully understand the optimization mode of CT images under the algorithm, and then, more perfect results and theoretical basis will be obtained. In conclusion, CT images based on the optimized reconstruction algorithm have high accuracy in the diagnosis of coronary heart disease, and the occurrence and development of coronary heart disease can be predicted by epicardial adipose tissue volume.

\section{Data Availability}

The data used to support the findings of this study are available from the corresponding author upon request.

\section{Conflicts of Interest}

The authors declare no conflicts of interest.

\section{References}

[1] SCOT-HEART Investigators, "CT coronary angiography in patients with suspected angina due to coronary heart disease (SCOT-HEART): an open-label, parallel-group, multicentre trial," Lancet, vol. 385, no. 9985, pp. 2383-2391, 2015 Jun 13.

[2] D. Andreini, R. Modolo, Y. Katagiri et al., "SYNTAX III REVOLUTION investigators. Impact of fractional flow reserve derived from coronary computed tomography angiography on heart team treatment decision-making in patients with multivessel coronary artery disease: insights from the SYNTAX III REVOLUTION trial," Circ Cardiovasc Interv, vol. 12, no. 12, Article ID e007607, 2019 Dec.

[3] P. D. Adamson, M. C. Williams, M. R. Dweck et al., "Guiding therapy by coronary CT angiography improves outcomes in patients with stable chest pain," Journal of the American College of Cardiology, vol. 74, no. 16, pp. 2058-2070, 2019 Oct 22. 
[4] X. Huo, H. M. Krumholz, X. Bai et al., "Effects of mobile text messaging on glycemic control in patients with coronary heart disease and diabetes mellitus: a randomized clinical trial," Circulation. Cardiovascular quality and outcomes, vol. 12, no. 9, Article ID e005805, 2019 Sep.

[5] M. C. Williams, J. H. Reid, G. McKillop et al., "Cardiac and coronary CT comprehensive imaging approach in the assessment of coronary heart disease," Heart, vol. 97, no. 15, pp. 1198-1205, 2011 Aug.

[6] M. Zanon, G. S. Pacini, V. V. S. de Souza et al., "Early detection of lung cancer using ultra-low-dose computed tomography in coronary CT angiography scans among patients with suspected coronary heart disease," Lung Cancer, vol. 114, pp. 1-5, 2017 Dec.

[7] M. Doris and D. E. Newby, "Coronary CT angiography as a diagnostic and prognostic tool: perspectives from the SCOTheart trial," Current Cardiology Reports, vol. 18, no. 2, p. 18, 2016 Feb.

[8] R. Yin, X. Tang, T. Wang et al., "Cardiac CT scanning in coronary artery disease: epicardial fat volume and its correlation with coronary artery lesions and left ventricular function," Experimental and Therapeutic Medicine, vol. 20, no. 4, pp. 2961-2968, 2020 Oct.

[9] M. Hu, Y. Zhong, S. Xie, H. Lv, and Z. Lv, "Fuzzy system based medical image processing for brain disease prediction," Frontiers in Neuroscience, vol. 15, Article ID 714318, 2021.

[10] N. N. Pandey, S. Sharma, P. Jagia, and S. Kumar, "Epicardial fat attenuation, not volume, predicts obstructive coronary artery disease and high risk plaque features in patients with atypical chest pain," British Journal of Radiology, vol. 93, no. 1114, Article ID 20200540, 2020 Oct 1.

[11] R. Khurana, A. Yadav, T. B. S. Buxi, J. P. S. Sawhney, K. S. Rawat, and S. S. Ghuman, "Correlation of epicardial fat quantification with severity of coronary artery disease: a study in Indian population," Indian Heart Journal, vol. 70, no. Suppl 3, pp. S140-S145, 2018 Dec.

[12] Y. Li, J. Zhao, Z. Lv, and J. Li, "Medical image fusion method by deep learning," International Journal of Cognitive Computing in Engineering, vol. 2, pp. 21-29, 2021.

[13] Q. Wu, Y. Chen, S. Chen, X. Wu, and W. Nong, "Correlation between adiponectin, chemerin, vascular endothelial growth factor and epicardial fat volume in patients with coronary artery disease," Experimental and Therapeutic Medicine, vol. 19, no. 2, pp. 1095-1102, 2020 Feb.

[14] H. Langet, C. Riddell, A. Reshef et al., "Compressed-sensingbased content-driven hierarchical reconstruction: theory and application to C-arm cone-beam tomography," Medical Physics, vol. 42, no. 9, pp. 5222-5237, 2015 Sep.

[15] Y. Ling, W. Meiniel, R. Singh-Moon, E. Angelini, J.-C. OlivoMarin, and C. P. Hendon, "Compressed sensing-enabled phase-sensitive swept-source optical coherence tomography," Optics Express, vol. 27, no. 2, pp. 855-871, 2019 Jan 21.

[16] Y. Nagayama, N. Nakamura, R. Itatani et al., "Epicardial fat volume measured on nongated chest CT is a predictor of coronary artery disease," European Radiology, vol. 29, no. 7, pp. 3638-3646, 2019 Jul.

[17] G. Milanese, M. Silva, R. E. Ledda et al., "Validity of epicardial fat volume as biomarker of coronary artery disease in symptomatic individuals: results from the ALTER-BIO registry," International Journal of Cardiology, vol. 314, pp. 20-24, 2020 Sep 1.
[18] B. J. Kim, J. G. Kang, S. H. Lee et al., "Relationship of echocardiographic epicardial fat thickness and epicardial fat volume by computed tomography with coronary artery calcification: data from the CAESAR study," Archives of Medical Research, vol. 48, no. 4, pp. 352-359, 2017 May. 